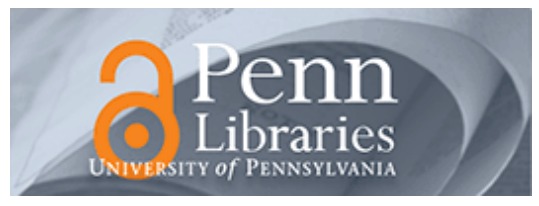

University of Pennsylvania

ScholarlyCommons

Statistics Papers

Wharton Faculty Research

$1-1-1986$

\title{
A View Of Reconstruction Tomography: XCT, ECT, NMRCT
}

Larry A. Shepp

University of Pennsylvania

Follow this and additional works at: https://repository.upenn.edu/statistics_papers

Part of the Computer Sciences Commons, and the Statistics and Probability Commons

\section{Recommended Citation}

Shepp, L. A. (1986). A View Of Reconstruction Tomography: XCT, ECT, NMRCT. SPIE Proceedings, 0671 http://dx.doi.org/10.1117/12.966671

This paper is posted at ScholarlyCommons. https://repository.upenn.edu/statistics_papers/402

For more information, please contact repository@pobox.upenn.edu. 


\title{
A View Of Reconstruction Tomography: XCT, ECT, NMRCT
}

\author{
Abstract \\ I survey the present status of three subfields of reconstruction tomography, $\mathrm{X}$-ray $\mathrm{CT}$, emission $\mathrm{CT}$, and \\ magnetic resonance $\mathrm{CT}$, and mention some new results and insights, as well as open problems. This is \\ for my Frontier in Imaging Science lecture at the IEEE Nuclear Science International Workshop on Physics \\ and Engineering of Computerized Multidimensional Imaging and Processing, April 2-4, 1986, Irvine, CA. \\ Disciplines \\ Computer Sciences | Statistics and Probability
}




\title{
A View of Reconstruction Tomography: XCT, ECT, NMRCT
}

\author{
L. A. Shepp \\ AT\&T Bell Laboratories \\ Murray Hill, NJ 07974
}

\begin{abstract}
I survey the present status of three subfields of reconstruction tomography, X-ray CT, emission CT, and magnetic resonance CT, and mention some new results and insights, as well as open problems. This is for my Frontier in Imaging Science lecture at the IEEE Nuclear Science International Workshop on Physics and Engineering of Computerized Multidimensional Imaging and Processing, April 2-4, 1986, Irvine, CA.
\end{abstract}

\section{§1. X-ray CT}

a. An industrial application: Medical CT is well documented, but some may not be aware that CT played a role recently in the space program. After two consecutive failures of the $4^{\prime}$ - rocket motors which are used to place communications satellites in geostationary orbit (after 18 consecutive successes) an expert panel was convened to try to determine the cause for the extinguishing of the motors during firing. (There are no O-rings in these motors!) They surmised that there is a possible delamination in the carbon exit cone of the motor and that this weakness can lead to the cone's disintegration and to the motor's extinguishing. Conventional ultrasound and radiographic images are unable to image such delaminations. A delamination is a curve or arc, of about $10^{\circ}$, in one transaxial plane of the cone, which is about $2 \mathrm{~cm}$ thick, in which one of the 60 laminar carbon-cloth layers making up the cone has a decreased density of at least $10 \%$. Thus we are trying to image a $10^{\circ}$ arc of an annulus of thickness of only $1 / 3 \mathrm{~mm}$. Industrial and medical CT scanners sample at about $2 \mathrm{~mm}$ intervals so it was thought that CT could not image the delamination either. However, as anyone in CT could have told them, the streak artifacts seen along tangents to discontinuities in objects, long a familiar problem in CT in patients with calcifications or bullets, etc. here proves actually helpful in enhancing such delaminations. A simulation clearly showed that even $1 \%$ delaminations can be (qualitatively) imaged and CT has become the method of choice for quality control of rocket exit cones. Despite this, there is a pressure from the insurance company to resume use of the stronger but heavier phenolic cones used in early launchings instead of carbon. The increased weight of the phenolic cones however leads to faster depletion of fuel available for keeping the satellite in geostationary orbit and hence to decreased lifetime of the satellite. For more details on this experience see [1].

b. High-speed $X$-ray $C T$ : While NMR has been making inroads into medical X-ray CT because of its many advantages in image quality, greater number of imaging parameters (density, $T_{2}$, flow, etc.), as well as dose, it has a serious problem, not yet overcome and perhaps unsolvable, of increased data-acquisition times. X-ray CT is much faster even in a sliceby-slice mode, and could be made even faster if many slices could be taken simultaneously. This could easily be done with a single pass of an X-ray tube provided that the problem of limited angle reconstruction can be solved. We have recently made some progress [2] in this direction with a simple new algorithm for limited angle CT.

Thus, suppose line integrals of $f(x, y)$ are known for lines $L$ which make an angle with the $x$-axis of at least $10^{\circ}$. While some older techniques attempt to extrapolate the missing views, and are subject to streak artifacts and noise sensitivity, we proceed in a direct and simple way as follows:

Define $g(x, y)=f(x, y / c)$ for a fixed $c<1$ and note that since $g$ is affinely related to $f$, any line integral of $g$ is also a line integral of $f$. It is easy to see that $c$ can be chosen, $c \approx 10$, so that the line integrals of $g$ are known for all lines $L$ which make an angle with the $x$-axis of at least $1^{\circ}$. But for $1^{\circ}$ it is reasonable and possible to use simple quadratures of Radon's formula to reconstruct $\boldsymbol{g}$ from these known projections. Finally we can obtain $f(x, y)=g(x, c y)$. For additional details we refer to [2]
Lest the reader feel swindled by the above argument, let me confess that the reconstructions of $f$ obtained with only $160^{\circ}$ as above are not as good as those with $180^{\circ}$ as in [3], but they are not so bad. Part of the problem may have to do with the fact that we are not using everything we may know about $g$ in the above argument. In particular we usually know for sure that $f$ vanishes off some circle in the $x-y$ plane. If this is the case, then $g$ vanishes off the corresponding ellipse after the transformation $(x, y) \rightarrow(x, y / c)$. If so, we don't know how to use this prior information about $g$.

\section{c. Prior information:}

Sometimes prior information in CT can play an enormous role. Recently a cute little problem came up-raised by B K P Horn of the MIT AI Laboratory-who is well-known in CT for his nice work on transforming Radon's theorem to new coordinates. Horn asked if it is known that $f(x, y)=0$ or 1 everywhere, whether the $x$ and $y$ projections determine $f$ uniquely if the $x$ and $y$ projections of $f$ are the same as those of the unit disk $D, x^{2}+y^{2} \leqslant 1$. It was very surprising to me to learn that only two projections of a disk, determine it uniquely among sets or among densities, if it is known that the density has only two values. Indeed, it almost seems that one could draw a counter-example. But the proof is easy: If $S$ is another set with same projections as $D$ then $S$ has the same area and moment of inertia as $D$ since $S$ and $D$ have the same $x$ and $y$ projections. But a disk of given area has least moment of inertia among all figures, uniquely, and we are done. For more details see [4]. Although this is a somewhat mathematical detour, there may be a moral here that one should not throw away real prior information lightly. Perhaps there are even better ways to do limited angle reconstruction.

\section{\$2. Emission CT}

Many groups have begun using the EM algorithm or variations on it to compute an approximate maximum likelihood estimate, $\hat{\lambda}$, of the true unknown emission density $\lambda$ as we suggested in [5], [6], [7]. While we were pleased at this interest in our work, it remains unclear whether or not emission CT has small enough error bars at its inherent low count rates to make it a useable and useful technique. Another problem, probably related, is that at high iteration numbers $n$ the EM estimate $\hat{\lambda}_{n}$ becomes snowy. Since the likelihood increments can be computed exactly, one can see that what is happening is that a small increase in likelihood is accompanied by a large increase in snow when $n$ is large. Some sort of smoothing seems to be required and one suggestion in this direction was made by Miller and Snyder [8] using the method of Grenander sieves. Another, very novel idea, was suggested by Grenander himself, with members of his group at Brown U. which will be discussed elsewhere by $D$. McClure [9], and is based on maximizing likelihood using an Ising-like model for $\lambda$ in which smoothness of $\lambda$ is built-in in a natural way and convergence takes place by a simulated annealing method.

Much effort has been spent in speeding up the E-M algorithm by using shortcuts [10], [11]. This effort continues and there have been several proposals for parallel computers for EM. On the other hand, these techniques must fail if the inherent error bars on $\hat{\lambda}$ are too large, at the realizable count rates. In double photon CT, single counts are discarded routinely, even though there are about 10 times as many singles counts in a planar-collimated one-ring experiment as doubles counts. Perhaps one reason that noone seriously considered using singles before is that it is clear that singles alone cannot reconstruct without focussed collimation since any circularly symmetric $\lambda$ would produce uniform counts in each detector and so different circularly symmetric $\lambda$ 's could not be discriminated even at infinite rates. But perhaps singles used along with doubles could help especially at low count rates. The EM algorithm [7] needs only relatively minor changes to accommodate singles as well as doubles: We take 
additional singles "tubes" for each detector and redefine $p(b, d)$ as in [7] using the attenuation density, assumed known. (We used a uniform attenuation density but could use say attenuation density obtained from $\mathrm{X}$-ray CT.) Here $d=$ single detector $d_{1}$ or a double-detector $\left(d_{1}, d_{2}\right)$. Linda Kaufman and I tried this experiment with $10^{7}$ counts generated by Monte-Carlo as in [7] of which about $10^{6}$ turned out to be doubles and the rest were singles, neglecting scatter.

I wish I could report that the singles helped. But unfortunately there was only slight improvement over doubles alone and we had to use 256 iterations to get about the same performance (or slightly better) than 32 iterations of doubles alone. Moreover by iteration 256 the reconstruction was again snowy, dashing any hopes that use of the singles would keep $\hat{\lambda}$ smooth in a natural way.

This experiment made me somewhat pessimistic, and in this mood I think it might be useful to try to determine some global notion of error bars for emission CT. Of course error bars on each pixel may be very large and one could still have a useful technology. For example some pixels in X-ray CT may be way off due to streaks or partial volume effects, etc. but the global X-ray CT image is still extremely useful. I do not believe in $L_{2}$ or $L_{p}$ metrics either. How does one do this meaningfully for emission CT? Perhaps one could show that emission CT with even $10^{6}$ counts can never be free of serious errors by some counting argument? Simulations by Miller and Snyder and others [8] indicate that $10^{5}$ count images have serious artifacts, apparently due just to randomness alone. On the other hand I think that [7] suggests that $10^{7}$ counts are adequate. How does one make these statements more quantitative?

\section{§3. NMR CT}

Whereas X-ray CT measures line integrals and emission CT measure counts in detectors or pairs of detectors, NMR CT measures the Fourie transform $\hat{f}$ of the spin density $f$ and reconstructs $f$ indirectly by using the Fourier inversion formula for reconstructing $f$. Thus despite our earlier claim [12] that one could regard NMR measurements as plane integrals of $f$, it is rather more direct and more accurate to represent the basic measured quantity in NMR as the approximate solution of the Bloch equation in the form

$$
S(t)=\int f \underset{\sim}{(x)} e^{i \int_{0}^{t} m(\underline{x}, s) d s} e^{-\frac{t}{T_{2}(x)}} d \underline{\sim}
$$

where $x$ may be 2 or 3 dimensional, $m(x, s)$ is the component of magnetization in the direction of the main magnetic field at $x$ at time $s$, and $T_{2}(x)$ is the $T_{2}$ damping coefficient at $x$ at time $t$ which causes the decay in the free-induction-decay signal $S$. Neglecting $T_{2}$ and taking the gradient field to be a ramp in direction $y$ with unit slope

$$
m(\underline{x}, s) \equiv \underline{x} \cdot \underline{y}
$$

where $\underline{y}$ is a fixed unit vector gives

$$
S(t)=\hat{f}(t \underline{y})
$$

where $\hat{f}$ is the Fourier transform of $f$. Choosing different directions for $y$ gives measurements of $\hat{f}(z)$ for $z-t y$ and so the Fourier transform of $\tilde{f}$ is approximated everywhere and $f$ can be determined by a quadrature of the Fourier inversion formula. Despite $T_{2}$ damping, inhomogeneities and non-linearities in (3.2) the method works very well and amazingly good reconstructions of $f$ are obtained. Data acquisition in NMR is slow because changing the direction of $y$ requires that the spins be allowed to relax and unless multiplane imaging with multiplexing of exciting anu relaxing spins takes place, long data acquisition times are obtained. Some attempts [13], [14] have been made at spiral or circular magnetization sequencing instead of $(3.2)$ so that during one spin cycle, $\hat{f}(z)$ can be sampled according to (3.1) at points of a curve which allows faster coverage of the transform parameter space. It is also possible to stop sampling $S(t)$ during the decay and to reenergize the spins with an echotype RF pulse in order to squeeze more information and speed up the data-acquisition. Finally, it is possible to supply less energy $\left(30^{\circ}\right.$ instead of $90^{\circ}$ pulse, say) so that equilibrium is reached more quickly.

To really separate effects and artifacts due to $T_{2}$, to nonuniform fields and gradients, to flow, to algorithms for inversion, to sampling schemes, to RF irregularities and to other parameters as well with real data is very difficult. On the other hand, computer simulation of (3.1) is not so easy either, especially with non-trivial $f$. Several attempts have been made [15], [16] but $f$ has always been taken to be a circle of constant density or a $\delta$-function or inexact calculations of $S(t)$ were used. However some cases are not hard. With the use of ellipses as in [3], we want to calculate (3.1) with $f$ constant inside the $n^{\text {th }}$ ellipse (or ellipsoid if $x$ is 3dimensional, but let's take the 2-dimensional case and write $\underset{\sim}{x}=(\tilde{x}, y)$ for simplicity). Then

(3.4) $S(t)=\sum_{n} S_{n}(t)=\sum_{n} d_{n} e^{-\frac{t}{T_{2, n}}} \iint_{-\infty}^{\infty} d x d y E_{n}(x, y) e^{i ø(x, y, t)}$

where

(3.5)

$$
\begin{aligned}
& E_{n}(x, y)=1 \text { if }\left(\left(x-\bar{x}_{n}\right)^{2} \cos \theta_{n}+\left(y-\bar{y}_{n}\right) \sin \theta_{n}\right)^{2} / a_{n}^{2}+\left(-\left(x-x_{n}\right) \sin \theta_{n}\right. \\
& \left.+\left(y-\bar{y}_{n}\right) \cos \theta_{n}\right)^{2} / b_{n}^{2} \leqslant 1
\end{aligned}
$$

and $E_{n}(x, y)=0$ otherwise, and $d_{n}$ is tissue density, $T_{2, n}$ is the $T_{2}$ value inside the $n^{\text {th }}$ ellipse, and

$\varnothing(x, y, t)=\gamma \int_{0}^{t} d s\left(x(s) G_{x}(s)+y(s) G_{y}(s)+x^{2}(s) B_{x x}(s)+x(s) y(s)\right.$

$\left.B_{x y}(s)+y^{2}(s) B_{g y}(s)\right)$

Here $G_{x}(t), G_{y}(t)$ are the $x$ and $y$ gradients at time $t$ and $B_{x x}(t), B_{x y}(t)$, and $B_{y y}(t)$ are the quadratic main field nonuniformity with arbitrary time dependence. The terms $x(t)$ and $y(t)$ allow for moving spins in that these terms give the position at time $t$ of the spin whose initial position at time 0 was $(x(0), y(0))=(x, y)$. In order to evaluate the integrals in (3.4) analytically, the terms $x(t)$ and $y(t)$ are restricted to be linear or quadratic functions of $x$ and $y$. The integrals in $x$ and $y$ are then complex Gaussian. The coefficients of these functions may have arbitrary time dependence, allowing simulation of complicated flow patterns. The phase $\varnothing(x, y, t)$ is a quadratic form in $x$ and $y$ and therefore by a series of $x-y$ coordinate transforms can be brought into a standard form. First, for each ellipse, suitable $x-y$ scaling, translation, and rotation is performed to map the ellipse onto the unit disk centered at the origin. Then an additional rotation is performed to eliminate the $B_{x y}^{(t)}$ term. Thus for each ellipse the signal equation (3.4) is reduced to a standard form given by

(3.7)

$$
\begin{aligned}
& S_{n}(t)=d_{n} e^{-t / T_{2 n}} e^{i \delta(t)} \int_{x^{2}+y^{2} \leqslant 1} d x d y \exp \left\{i \left(\alpha_{n}(t) x+A_{n}(t) x^{2}\right.\right. \\
& \left.+\beta_{n}(t) y+B_{n}(t) y^{2}\right\}
\end{aligned}
$$

The variables $\alpha_{n}(t), A_{n}(t), \beta_{n}(t), B_{n}(t)$ and $\delta_{n}(t)$ are linear combinations of the integrated gradient, integrated nonuniformity, and ellipse parameters. Generally, $\alpha_{n}(t)$ and $\beta_{n}(t)$ are most strongly dependent on the integrated gradient terms $\int G_{x} d t$ and $\int G_{y} d t$ while $A_{n}(t)$ and $B_{n}(t)$ are most strongly dependent on the integrated nonuniformity terms, $\int B_{x x} d t, \int B_{x y} d t$, and $\int B_{y y} d t$. Thus the basic form of the signal simulation integral is

$$
(3.8) I=I(\alpha, A, \beta, B)=\int_{x^{2}+y^{2} \leqslant 1} \int_{1} d x d y \exp \left\{i\left(\alpha x+A x^{2}+\beta y+B y^{2}\right)\right\}
$$


If there are no nonlinearities or nonuniformities then $A=B=0$ in (3.8) which allows the integrals over ( $x, y)$ in $(3.8)$ to be extended to $(-\infty, \infty)$. and $I$ can be written simply in terms of the Bessel function $J_{1}$. In this way This double integral over $x$ and $y$ is a complex Gaussian or Fresnel the effect of $T_{2}$ can be simply and exactly simulated on a realistic phantom integral and can be done explicitly. The result is in closed form (since $J_{1}$ is readily available in most numerical computer packages). Thus for $A=B=0$,

(3.9) $I(\alpha, 0, \beta, 0)=2 \pi \int_{0}^{1} r d r \int_{0}^{2 \pi} d \theta e^{i \sqrt{\alpha^{2}+\beta^{2}} r \cos \theta}=\frac{2 \pi J_{1}\left(\sqrt{\alpha^{2}+\beta_{2}}\right)}{\sqrt{\alpha^{2}+\beta^{2}}}$ (3.11)

For $A B \neq 0$, the two-dimensional integral (3.8) can be reduced to a onedimensional integral by a neat trick. We introduce a third integral

$$
\int_{-\infty-i \epsilon}^{\infty-i \epsilon} d u\left(\frac{e^{i u}-e^{-i u}}{2 \pi i u}\right) e^{i u\left(x^{2}+y^{2}\right)}= \begin{cases}1 & x^{2}+y^{2} \leqslant 1 \\ 0 & \text { otherwise }\end{cases}
$$$$
I=\frac{1}{4} \int_{-\infty-i \epsilon}^{\infty+i \epsilon} d u \frac{e^{i u}-e^{-i u}}{i u} \exp \left(-\frac{i}{4}\left[\frac{\alpha^{2}}{A-u}+\frac{\beta^{2}}{B-u}\right]\right)((A-u)(B-u))^{-1 / 2}
$$

The latter integral can be reduced further and evaluated asymptotically for various values of the parameter by shifting the contour of integration in the $u$-plane since the integrand is analytic. This has been carried out in [17] and has produced very useful and useable results, which will be reported elsewhere.

\section{FIGURE CAPTIONS}

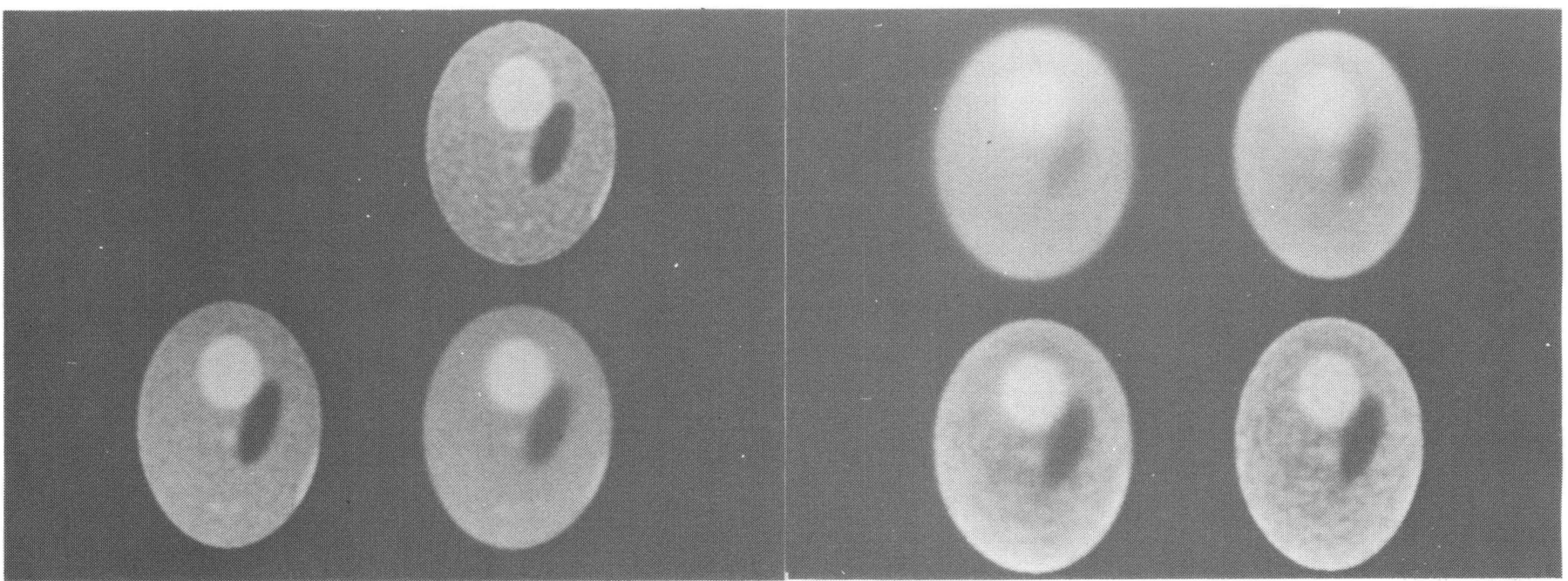

Figure 1. E-M reconstruction with $n$ iterations of L. Kaufman's linesearch speed-up [10]. $n=8$ lower right, $n=16$ lower left, $n=32$ upper right (this is $\sim 500$ E-M steps). $10^{7}$ counts, doubles alone. Assumes no attenuation as in $[5,6]$.
Figure 3. $\mathrm{E}-\mathrm{M}$ with $\sim 10^{6}$ doubles and $\sim 10^{7}$ singles. Attenuation corrected by E-M as in Fig. 2. 32, 64, 256, 128 E-M steps clockwise from upper left. Note that 256 iterations is again noisy (unfortunately). Only slightly, if at all, better than Fig. 2 .

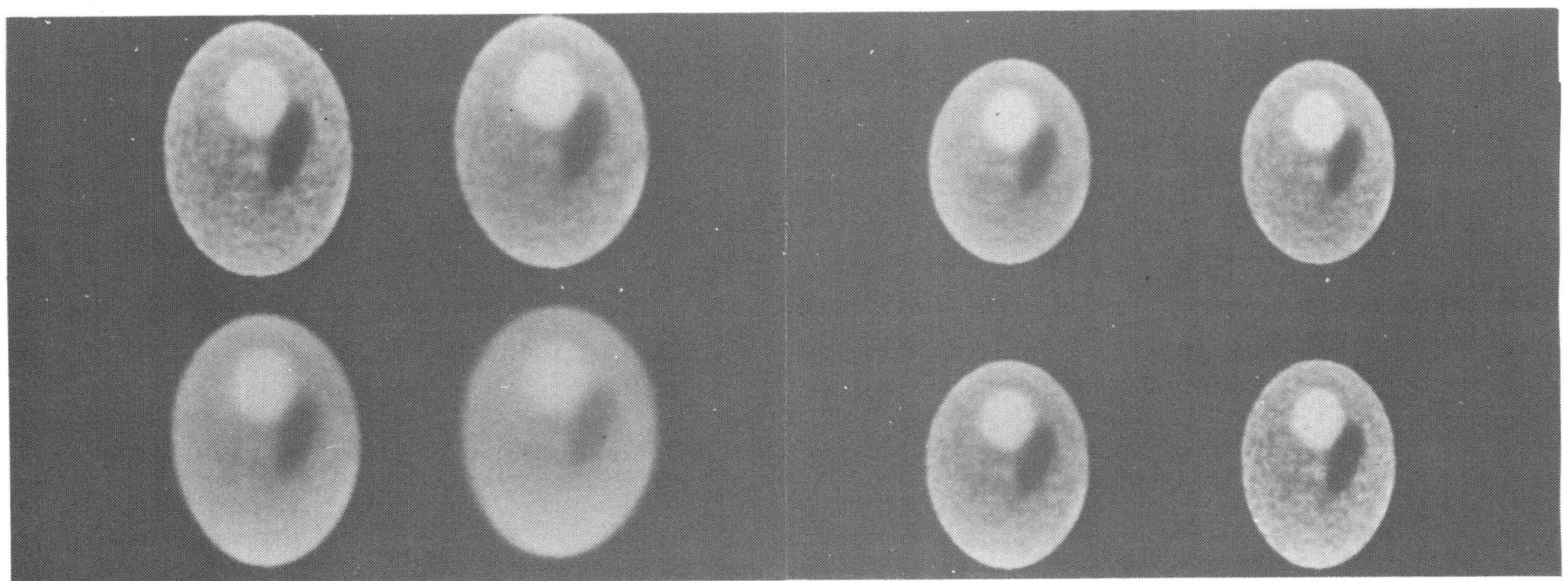

Figure 2. E-M with doubles alone, corrected for attenuation by using $p(b, d)$ determined by using (uniform) attenuation density. $10^{6}$ counts. Iterations: $32,16,8,16$ of E-M, clockwise from upper left.
Figure 4. Direct comparison of $10^{6}$ doubles, $10^{6}$ doubles $+10^{7}$ singles on same picture. Iterations: 128,256 of $10^{7}$ singles $+10^{6}$ doubles on top from left as in Fig. 3. Iterations: 16, 32 of $10^{6}$ doubles on bottom from left as in Fig. 2. 


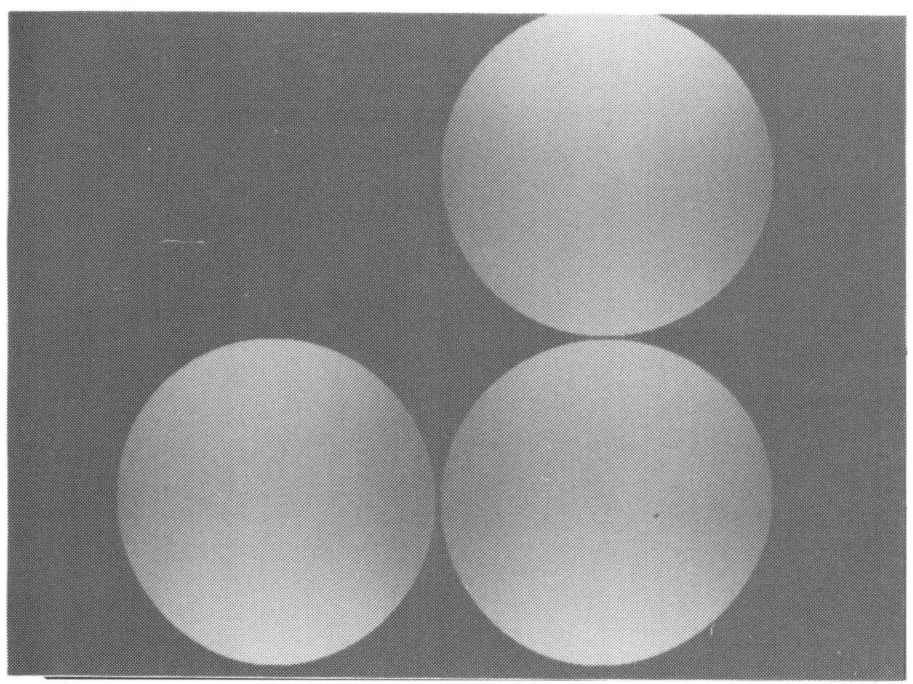

Figure 5. $10^{7}$ singles alone. $64,128,256$ iterations. Clearly useless, as should be expected since there are only 128 different tubes to register the $10^{7}$ singles counts because there are only 128 different detectors. The increased counts at the top and bottom are no doubt due to the shape of the phantom.
[3] Shepp, L. A. and Logan B. F., The Fourier reconstruction of a head section, IEEE Trans. Nucl. Sci. NS-21, 1974, 21-43.

[4] Fishburn P C, Reeds J A, and Shepp L A. Unique reconstruction of sets from projections, Ms. in preparation.

[5] Shepp L A and Vardi Y, Maximum likelihood reconstruction for emission tomography, IEEE Trans. Med. Imag. Vol. MI-1, 1982, 113-122.

[6] Vardi Y, Shepp L A, Kaufman L, A statistical model for position emission tomography, JASA, 80, 1985, 8-37.

[7] Shepp L A, Vardi Y, Ra J B, Hilal S K, Cho Z H, Maximum likelihood PET with real data, IEEE Trans Nucl Sci, NS-31, 1984, 910-913.

[8] Miller M I and Snyder, D. The use of sieves to stabilize images produced with the EM algorithm for emission tomography, IEEE Trans Nucl. Sci. NS-32, 1985, 3864-3872.

[9] McClure, D. Personal correspondence.

[10] Kaufman, L. Implementing and accelerating the EM algorithm for PET, to appear.

[11] Levitt R M and Muehllehner, G., Accelerated iterative reconstruction for PET based on the EM algorithm, Ms. June 1985.

[12] Shepp, L A, Computerized tomography and NMR, J Comp Asst. Tomo., 4, 1980, 94-107.

[13] Buonocore, M. Personal correspondence.

[14] Ahn, C B, Reiv, C Y, Kim J H and Cho Z H, New high-speed spiral-scan echo planar imaging I. Ms.

[15] Lai C M, Reconstructing NMR images under magnetic fields with large inhomogeneities, J. Phys. E. Sci. Instr. 15, 1982, 1093-1100.

[16] O'Donnell, M and Edelstein, W. A., NMR imaging in the presence of magnetic field inhomogeneities and gradient field nonlinearities, Med. Phys. 12, 1985, 20-26.

[17] Buonocore, M H, Perlmutter, R J, Kirk, G L, and Shepp L A, A method for simulation in NMR imaging. Ms in preparation. 